\title{
EMBEDDING A SEMIGROUP OF TRANSFORMATIONS
}

\author{
J. S. V. SYMONS \\ (Received 27 November 1973) \\ Communicated by T. E. Hall
}

Let $X$ be an arbitrary set and $\theta$ a transformation of $X$. One may use $\theta$ to induce an associative operation in $\mathscr{T}_{X}$, the set of all mappings of $X$ to itself as follows:

$$
\alpha * \beta=\alpha \theta \beta \quad\left(\alpha, \beta \in \mathscr{T}_{X}\right) .
$$

We denote the resulting semigroup by $\left(\mathscr{T}_{X} ; \theta\right)$ Magill (1967) introduced this structure and it has been studied by Sullivan and by myself.

Sullivan asks when $\left(\mathscr{T}_{X} ; \theta\right)$ can be embedded in $\left(\mathscr{T}_{X}, 0\right)$, the full transformation semigroup under composition. He shows that if $X$ is finite this can be done if and only if $\theta$ is a permutation of $X$ and that any embedding (an isomorphism perforce) is of the form

$$
\alpha \rightarrow g^{-1} \theta \alpha g \quad\left(\alpha \in \mathscr{T}_{X}\right)
$$

where $g$ is a permutation of $X$. The infinite case is left open. The purpose of this note is to prove the following.

THEOREM 1. If $X$ is infinite then any $\left(\mathscr{T}_{X}, \theta\right)$ may be embedded in $\left(\mathscr{T}_{X}, 0\right)$.

Proof. Let $X=X_{E} \cup X_{0}$ where $X_{0}$ and $X_{0}$ are disjoint and of the same cardinality, necessarily that of $X$. Select bijections $g$ and $h$ such that

$$
h: X \rightarrow X_{E} \text { and } g: X \rightarrow X_{0} \text {. }
$$

For $\alpha$ in $\mathscr{T}_{X}$ we define $\alpha \phi$ as follows

$$
\begin{aligned}
x \alpha \phi & =x h^{-1} \alpha g & & \left(x \in X_{E}\right) \\
& =x g^{-1} \theta \alpha g & & \left(x \in X_{0}\right) .
\end{aligned}
$$

It is clear that the first part of the definition guarantees that $\alpha \rightarrow \alpha \phi$ is one to one. Observe that $\alpha \phi: X \rightarrow X_{g}=X_{0}$. It follows that for $\alpha$ and $\beta$ in $\mathscr{T}_{X}$ we have that $\alpha \phi \beta \phi=\alpha \phi g^{-1} \theta \beta g$. Thus if $x$ is in $X_{E}$, 


$$
x \alpha \phi \beta \phi=x h^{-1} \alpha g \cdot g^{-1} \theta \beta g=x h^{-1} \alpha \theta \beta g=x\left(\alpha^{*} \beta\right) \phi
$$

while if $x$ is in $X_{0}$,

$$
x \alpha \phi \beta \phi=x g^{-1} \theta \alpha g \cdot g^{-1} \theta \beta g=x g^{-1} \theta \alpha \theta \beta g=(x * \beta) \phi .
$$

It follows that $(\alpha * \beta) \phi=\alpha \phi \beta \phi$, as required.

The classification of the embeddings of $\left(\mathscr{T}_{X} ; \theta\right)$ in $\left(\mathscr{T}_{X}, 0\right)$ is extremely difficult. Partial results have been obtained. We shall describe our most pleasant result in this direction.

We call a transformation semigroup $S \subseteq \mathscr{T}_{X}$ irreducible if the set

$$
x S=\{x \alpha ; \alpha \in S\}
$$

coincides with $X$ for each $x$ in $X$. Further, we shall say than an embedding $\phi$ of $\left(\mathscr{T}_{X} ; \theta\right)$ in $\left(\mathscr{T}_{X}, 0\right)$ is irreducible if $\mathscr{T}_{X} \phi$ is irreducible.

THEOREM 2. Any irreducible embedding of $\left(\mathscr{T}_{X} ; \theta\right)$ in $\left(\mathscr{T}_{X}, \circ\right)$ is of the form

$$
\alpha \rightarrow g^{-1} \theta \alpha g \quad\left(\alpha \in \mathscr{T}_{X}\right)
$$

for some fixed permutation $g$ of $X$.

Proof. Assume $\phi$ is such an embedding and let $\kappa=\kappa_{x}$ denote the constant function in $\mathscr{T}_{x}$ with range $x$. We choose $y$ in the range of $\kappa \phi$ and consider $y(\kappa \phi)^{-1}$. If $z$ is any member of this latter set then for any $\alpha$ in $\mathscr{T}_{X}$

$$
(z \alpha \phi) \kappa \phi=z(\alpha \kappa) \phi=z \kappa \phi=y .
$$

This shows that $y(\kappa \phi)^{-1}$ is invariant under $\mathscr{T}_{X} \phi$ and hence, by irreducibility, coincides with $X$. This shows that $\phi$ maps constants to constants from which follows

$$
\kappa_{x} \phi=\kappa_{x g} \quad(x \in X)
$$

where $g$ is an injective transformation of $X$. But then for each $\alpha$

$$
\kappa_{x \theta \times g}=\left(\kappa_{x}^{*} \alpha\right) \phi=\kappa_{x} \phi \alpha \phi
$$

which implies $\theta \alpha g=g \alpha \phi$. Thus $X g \alpha \phi \subseteq X g$, and this is contrary to irreducibility unless $g$ is onto. In this case $g$ permutes $X$ and $\alpha \phi=g^{-1} \theta \alpha g$, as required.

It is clear that $\phi$ above is an embedding if and only if $\theta$ is onto $X$. Hence we have the following:

Corollary. It is possible to irreducibly embed $\left(\mathscr{T}_{X} ; \theta\right)$ in $\left(\mathscr{T}_{X}, 0\right)$ if and only if $\theta$ is onto. 


\section{References}

K. D. Magill, Jr. (1967), 'Semigroup structures for familes of functions, I. Some homomorphism theorems', J. Austral. Math. Soc. 7, 81-94.

R. P. Sullivan (to appear), 'Generalized partial transformation semigroups', J. Austral. Math. Soc. J. S. V. Symons (to appear), 'On a generalization of the transformation semigroup', J. Austral. Math. Soc.

J. S. V. Symons (1973), Automorphisms of transformation semigroups (Ph.D. thesis, University of Western Australia, 1973).

Monash University

Clayton 3168, Australia. 